INPLASY

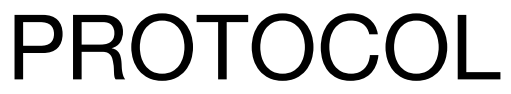

To cite: Currier et al. The Influence of Resistance Exercise Training Prescription Variables on Muscle Mass, Muscle Strength, and Physical Function in Healthy Adults: An Umbrella Review. Inplasy protocol 202220028. doi: 10.37766/inplasy2022.2.0028

Received: 10 February 2022

Published: 10 February 2022

Corresponding author: Stuart Phillips

phillis@mcmaster.ca

Author Affiliation:

McMaster University.

Support: None.

Review Stage at time of this submission: Preliminary searches.

Conflicts of interest: None declared.

\section{The Influence of Resistance Exercise Training Prescription Variables on Muscle Mass, Muscle Strength, and Physical Function in Healthy Adults: An Umbrella Review}

Currier, BS1; Mcleod, JC2; Phillips, SM³.

Review question / Objective: To determine how resistance training prescription variables (load, sets, frequency, time under tension, etc) affect muscle mass, muscle strength, and physical function in healthy adults.

Condition being studied: To determine how resistance training prescription variables (load, sets, frequency, time under tension, etc) affect muscle mass (hypertrophy), muscle strength, and physical function in healthy adults.

Information sources: OVID MEDLINE, SPORTDiscus, Web of Science.

INPLASY registration number: This protocol was registered with the International Platform of Registered Systematic Review and Meta-Analysis Protocols (INPLASY) on 10 February 2022 and was last updated on 10 February 2022 (registration number INPLASY202220028).

\section{INTRODUCTION}

Review question / Objective: To determine how resistance training prescription variables (load, sets, frequency, time under tension, etc) affect muscle mass, muscle strength, and physical function in healthy adults.

Rationale: We do not fully understand how resistance training (RT) variables affect muscle growth and strength. Knowing the 
answer to this question would assist in programming $R T$ to develop these characteristics.

Condition being studied: To determine how resistance training prescription variables (load, sets, frequency, time under tension, etc) affect muscle mass (hypertrophy), muscle strength, and physical function in healthy adults.

\section{METHODS}

Search strategy: OVID MEDLINE Epub Ahead of Print, In-Process \& Other NonIndexed Citations, Ovid MEDLINE(R) Daily and Ovid MEDLINE(R) 1946 to Present (December 2021); SPORTDiscus (December 2021); Web of Science (December 2021).

Participant or population: Adults (>18yr).

Intervention: Resistance training.

Comparator: non-RT group, alternative RT condition (i.e., differing variables according to the FITT principle)

Study designs to be included: Systematic reviews of RT.

Eligibility criteria: Systematic reivews.

Information sources: OVID MEDLINE, SPORTDiscus, Web of Science.

Main outcome(s): Muscle mass: fat-free mass (FFM) and bone-fat-free mass (or lean mass if FFM was not available; FFM; measured by dual-energy X-ray absorptiometry (DXA), hydro densitometry, whole-body air plethysmography (BodPod), or bioelectrical impedance); muscle fibre cross-sectional area (CSA; measured in any fibre subtype (I, Ila, and/or IIx) obtained from skeletal muscle biopsies using microscopy); whole muscle CSA measured by (Ultrasound, MRI and/or CT); whole muscle volume (Ultrasound, MRI and/or CT); or whole muscle thickness (Ultrasound, MRI and/or CT) Muscle strength: one-repetition-maximum strength (1RM; measured by any 1RM strength test) and maximum voluntary contraction (MVC; measured by both isokinetic and/or isometric contractions using a dynamometer with any muscle group/ action). Physical Function: Timed up and go, 6-minute walk test, any measure of walking speed (25-foot walk test), Sit to stand test, short performance physical battery, berg balance scale. Measures of effect.

Additional outcome(s): None.

Data management: All data are stored or managed on the PI and CO-I's personal computers. Extracted data are curated and shared on cloud-based databases.

Quality assessment / Risk of bias analysis: - A MeaSurement Tool to Assess Systematic Reviews (AMSTAR 2)

- The Grading of Recommendations Assessment Development and Evaluation (GRADE).

Strategy of data synthesis: Summary of results across included systematic reviews will be separated by outcome domain (muscle mass, strength, and physical function) and intervention/comparator type (resistance exercise training versus nonexercise control group, sets, load, frequency, time under tension, etc) alongside the methodological quality of the review, number of trials included in the relevant analysis and sample size. The overall effectiveness of each intervention will be based on the total number of participants affected positively across the relevant systematic reviews.

Subgroup analysis: If an adequate amount of systematic reviews are available, then we will apply the aforementioned data synthesis strategy on two sub-groups of our total sample size, based on age: young adults (18 - 54 years old), and older adults (>= 55 years old).

Sensitivity analysis: None-planned.

Language: English.

Country(ies) involved: Canada. 
Other relevant information: Inclusion Criteria:

- Humans $\geq 18$ years old

- Generally healthy (no disease condition indicated)

- Community-dwelling adults

Exclusion Criteria:

- Humans $<18$ years old

- Persons with or at risk for comorbidities (e.g., cardiovascular disease, type II diabetes, type I diabetes, cancer, peripheral artery disease, osteoarthritis, etc.).

- Persons that are injured (e.g., MSK related fracture and/or repair).

- Explicitly mentions obese and/or overweight participants.

- Individuals that are hospitalized (inpatient/outpatient/rehabilitation, etc).

- Individuals living in long-term care homes.

- Animal studies (e.g., murine models)

Inclusion Criteria:

-Resistance exercise training compared with a non-exercise control group OR resistance exercise training variable is manipulated (e.g., sets, load, frequency, time under tension, etc) and compared with another group performing resistance exercise training.

Exclusion:

- Resistance exercises training prescription variable not manipulated between groups OR resistance exercise training not compared to a non-exercise control group

- Groups were exposed to different conditions in addition to resistance exercise training (e.g., nutritional supplementation)

Types of study to be included

- Systematic Reviews (Narrative Summaries, Meta-Analyses, and/or Network Meta Analyses)

Exclusion Criteria:

- Scoping or narrative reviews that do not include critical appraisal of primary studies.

- Systematic reviews not published in English.

- Primary research (e.g., Randomizedcontrolled trials, case report, case-control, cohort study)"

Keywords: muscle mass, hypertrophy, strength, resistance training.
Dissemination plans: Presentations at academic meetings, written up for publication in a scholarly journal.

Contributions of each author:

Author 1 - Brad Currier - All phases: idea inception, search, data screening, data synthesis, writing, and editing.

Email: currierb@mcmaster.ca

Author 2 - Jonathan Mcleod - All phases: idea inception, search, data screening, data synthesis, writing, and editing.

Author 3 - Stuart Phillips - All phases: idea inception, search, data screening, data synthesis, writing, and editing.

Email: phillis@mcmaster.ca 